CRYSTALLOGRAPHIC COMMUNICATIONS

ISSN 2056-9890

Received 8 October 2017

Accepted 17 October 2017

Edited by W. T. A. Harrison, University of Aberdeen, Scotland

Keywords: crystal structure; halogenobismuthates; benzotriazole; Hirshfeld surface analysis.

CCDC reference: 1580458

Supporting information: this article has supporting information at journals.iucr.org/e

\section{Synthesis, crystal structure and Hirshfeld surface analysis of a polymeric bismuthate(III) halide complex, $\left(\mathrm{C}_{6} \mathrm{H}_{6} \mathrm{~N}_{3}\right)_{2}\left[\mathrm{BiCl}_{5}\right] \cdot 2 \mathrm{H}_{2} \mathrm{O}$}

\author{
Chaima Boukoum, ${ }^{\mathrm{a} *}$ Zouhaier Aloui, $^{\mathrm{a}}$ Valeria Ferretti ${ }^{\mathrm{b}}$ and Sonia Abid $^{\mathrm{a}}$ \\ áaboratoire de Chimie des Matériaux, Faculté des Sciences de Bizerte, 7021 Zarzouna Bizerte, Tunisia, and ${ }^{\mathbf{b}}$ Department \\ of Chemical and Pharmaceutical Sciences, Centre for Structural, Diffractometry, University of Ferrara, Via L. Borsari 46, I- \\ 44121 Ferrara, Italy. ${ }^{*}$ Correspondence e-mail: ch.boukoum@gmail.com
}

The synthesis and the crystal structure of a new halide-bridged polymer, namely catena-poly[bis(1,2,3-benzotriazolium) [[tetrachloridobismuth(III)]- $\mu$-chlorido] dihydrate], $\left\{\left(\mathrm{C}_{6} \mathrm{H}_{6} \mathrm{~N}_{3}\right)_{2}\left[\mathrm{BiCl}_{5}\right] \cdot 2 \mathrm{H}_{2} \mathrm{O}\right\}_{n}$ are reported. The structure comprises polyanionic zigzag chains of formula $\left[\left(\mathrm{BiCl}_{5}\right)^{2-}\right]_{n}$ running along the $c$-axis direction. The 1,2,3-benzotriazolium cations are linked between these polymer chains, via the water molecules, giving rise to left- and right-handed helical chains. Hirshfeld surface analysis and fingerprint plots were used to decode the intermolecular interactions in the crystal network and determine the contribution of the component units for the construction of the three-dimensional architecture.

\section{Chemical context}

Bismuth-halide complexes are of contemporary interest because of their structural diversity and numerous promising physical properties such as dielectric, ferroelectric, ferroelastic, non-linear optical and thermochromism (Bator et al., 1997; Bednarska-Bolek et al., 2000; Sobczyk et al., 1997; Bator et al., 1998). Generally, in these compounds, the $\mathrm{Bi} X_{6}$ octahedra may join to form discrete (i.e. mononuclear) or extended (i.e. polynuclear) inorganic networks of corner-, edge-, or face-sharing octahedra, leading to an extensive family of bismuth halogenoanions (Jakubas, 1986; Jakubas et al., 1988, 1995). A variety of organic cations, ring shaped or linear, have a strong impact on the arrangements of $\mathrm{Bi} X_{6}$ octahedra and the formation of hydrogen bonds (Dammak et al., 2015; Elfaleh \& Kamoun, 2014). This class of compounds has also attracted much attention in the field of crystal engineering over the last decade on account of their capability for the creation of extended architectures via intermolecular non-covalent binding interactions. (i.e. hydrogen bonding, ionic and $\pi-\pi$ stacking interactions; Belter \& Fronczek, 2013; Thirunavukkarasu et al., 2013; Aloui et al., 2015).

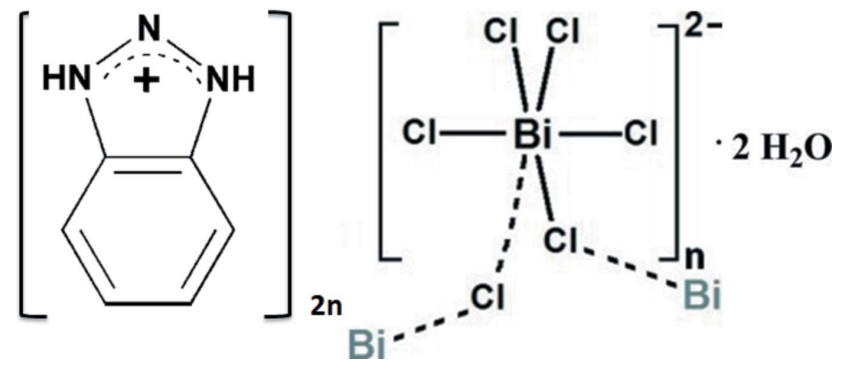




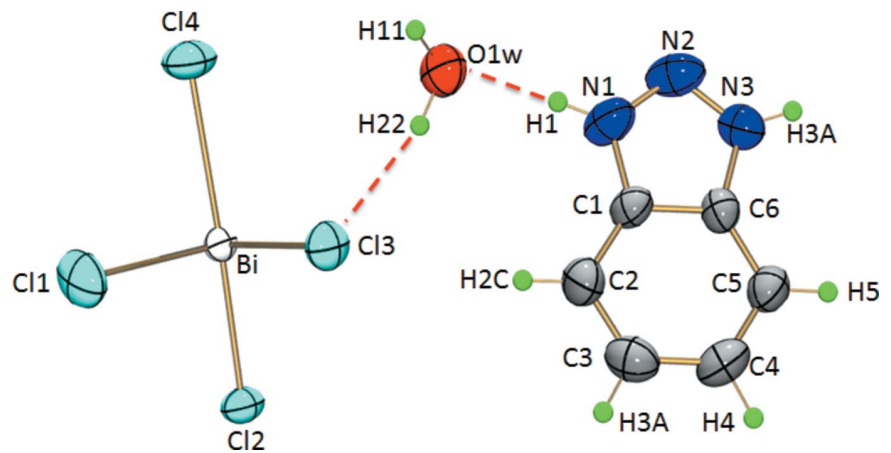

Figure 1

The asymmetric unit of (I) showing 50\% displacement ellipsoids.

As part of our studies in this area, we chose benzotriazole, which is an aromatic heterocyclic base with three protonatable nitrogen atoms, as the organic cation.

\section{Structural commentary}

The single-crystal $\mathrm{X}$-ray diffraction analysis shows that the title compound $\left[\mathrm{C}_{6} \mathrm{H}_{6} \mathrm{~N}_{3}\right]_{2}\left[\mathrm{BiCl}_{5}\right] \cdot 2 \mathrm{H}_{2} \mathrm{O}$, (I), crystallizes in the
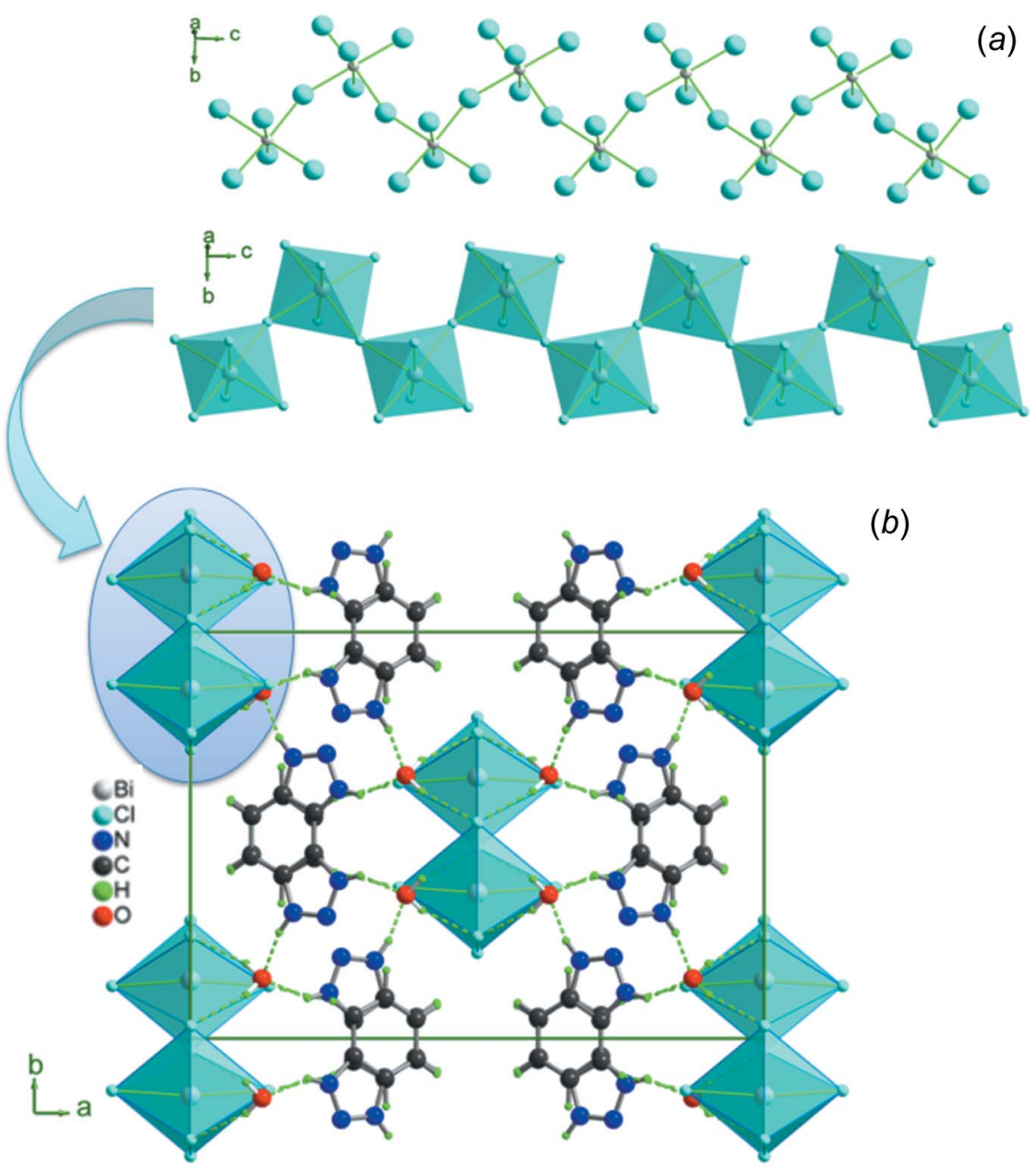

Figure 2

(a) View of the $\left[\left(\mathrm{BiCl}_{5}\right)^{2-}\right]_{n}$ polyanionic zigzag chains in (I) along the $c$-axis direction. (b) Projection along the $c$ axis of the structure of (I). (b)

Table 1

Selected bond lengths $(\AA)$.

\begin{tabular}{llll}
\hline $\mathrm{Bi} 1-\mathrm{Cl} 1$ & $2.669(3)$ & $\mathrm{Bi} 1-\mathrm{Cl} 4^{\mathrm{i}}$ & $2.757(4)$ \\
$\mathrm{Bi} 1-\mathrm{Cl} 2$ & $2.545(3)$ & $\mathrm{Bi} 1-\mathrm{Cl} 4$ & $2.856(4)$ \\
$\mathrm{Bi} 1-\mathrm{Cl} 3$ & $2.674(4)$ & & \\
\hline
\end{tabular}

Symmetry code: (i) $-x+1,-y+1, z-\frac{1}{2}$.

non-centrosymmetric space group $C m c 2_{1}$ and the asymmetric unit comprises one $\mathrm{Bi}^{3+}$ cation, four chlorine atoms, one water molecule and one benzotriazolium cation (Fig. 1). The bismuth atom is six-coordinated by four distinct chlorine atoms $(\mathrm{Cl} 1, \mathrm{Cl} 2, \mathrm{Cl} 3, \mathrm{Cl} 4)$. The $\mathrm{Bi}-\mathrm{Cl}$ bond lengths (Table 1) vary from $2.545(3)$ to $2.674(4) \AA(\Delta \mathrm{Bi}-\mathrm{Cl}=0.129 \AA)$ and 2.757 (4) to 2.856 (4) $\AA$ ( $\Delta \mathrm{Bi}-\mathrm{Cl}=0.099 \AA$ ) for non-bridging and bridging $\mathrm{Cl}$ atoms, respectively, which are comparable with values found in $\left\{\left(\mathrm{C}_{2} \mathrm{H}_{7} \mathrm{~N}_{4} \mathrm{O}\right)_{2}\left[\mathrm{BiCl}_{5}\right]_{n}\right.$ (Ferjani et al., 2012) and $\left[\mathrm{NH}_{3}\left(\mathrm{CH}_{2}\right)_{6} \mathrm{NH}_{3}\right] \mathrm{BiCl}_{5}$ (Ouasri et al., 2013). The $\mathrm{Cl}-\mathrm{Bi}-$ $\mathrm{Cl}$ bond angles in (I) range from 85.93 (17) to $91.88(13)^{\circ}$ $\left(\triangle \mathrm{Cl}-\mathrm{Bi}-\mathrm{Cl}=5.95^{\circ}\right)$ and are less distorted than those observed in $\left[\mathrm{NH}_{3}\left(\mathrm{CH}_{2}\right)_{6} \mathrm{NH}_{3}\right] \mathrm{BiCl}_{5}$ and $\left[\mathrm{H}_{2}\right.$ mdap $]\left[\mathrm{BiCl}_{5}\right]$ (Ouasri et al., 2013; Wang et al., 2017).

In the extended structure of (I), adjacent $\mathrm{BiCl}_{6}$ octahedra are connected through $\mathrm{Cl} 4$ and $\mathrm{Cl} 4{ }^{\mathrm{iii}}$ so as to form $\left[\left(\mathrm{BiCl}_{5}\right)^{2-}\right]_{n}$ polyanionic zigzag chains propagating along the caxis direction, with the shortest intrachain $\mathrm{Bi} \cdot \cdots \mathrm{Bi}$ distance of 5.508 (1) $\AA$ and a $\mathrm{Cl} 4-\mathrm{Bi}-\mathrm{Cl} 4^{\mathrm{ii}}$ angle of 89.61 (3) ${ }^{\circ}$ (Fig. 2) The overall negative charges of the resulting polymers are counterbalanced by the protonated 1,2,3benzotriazolium cations (Fig. 2b). As usual, this aromatic amine is protonated at the $\mathrm{N} 3$ atom and the $\mathrm{C}-\mathrm{C}, \mathrm{N}-\mathrm{N}$ and $\mathrm{C}-\mathrm{N}$ bond lengths vary from 1.358 (18) to $1.402(15), 1.293(15)$ to 1.308 (15) $\AA$ and 1.364 (16) to 1.370 (15) $\AA$, respectively, which agree well with those observed in bis(1,2,3-benzotriazolium) sulfate dihydrate (Randolph et al., 2013) and benzotriazolium picrate (Zeng et al., 2011).

\section{Supramolecular features}

The heterocyclic cations alternately bridge the water molecules (O1W) via $\mathrm{N}-\mathrm{H} \cdots \mathrm{O}$ hydrogen bonds, forming (benzo-OW $)_{n}$ helical chains in a rightand left-handed sequence extending along the $c$-axis direction (Table 2, Fig. 2). The phenyl rings of adjacent chains are alternately stacked in a parallel-displaced face-to-face arrangement (Fig. 3), with centroid-centroid distances of 3.8675 (1) $\AA$ and an interplanar spacing of $1.13 \AA$. The anionic 
Table 2

Hydrogen-bond geometry $\left(\AA,^{\circ}\right)$.

\begin{tabular}{lllll}
\hline$D-\mathrm{H} \cdots A$ & $D-\mathrm{H}$ & $\mathrm{H} \cdots A$ & $D \cdots A$ & $D-\mathrm{H} \cdots A$ \\
\hline $\mathrm{N} 1-\mathrm{H} 1 \cdots \mathrm{O} 1 W$ & 0.86 & 2.08 & $2.891(17)$ & 157 \\
$\mathrm{~N} 3-\mathrm{H} 3 A \cdots \mathrm{O} 1 W^{\text {ii }}$ & 0.86 & $2.00(2)$ & $2.767(18)$ & 148 \\
$\mathrm{O} 1 W-\mathrm{H} 11 \cdots \mathrm{Cl} 4^{\text {iii }}$ & $0.89(11)$ & $2.47(11)$ & $3.306(13)$ & $157(9)$ \\
$\mathrm{O} W-\mathrm{H} 22 \cdots \mathrm{Cl} 3$ & $0.90(12)$ & $2.38(12)$ & $3.268(14)$ & $169(11)$ \\
$\mathrm{C}^{-\mathrm{H} 5} \cdots \mathrm{Cl}{ }^{\text {iv }}$ & 0.93 & 2.73 & $3.603(14)$ & 157 \\
\hline
\end{tabular}

Symmetry codes: (ii) $\quad-x+\frac{3}{2},-y+\frac{1}{2}, z+\frac{1}{2}$; $\quad$ (iii) $\quad-x+1,-y+1, z+\frac{1}{2}$; (iv) $x+\frac{1}{2}, y-\frac{1}{2}, z+1$.

and cationic chains are further assembled into a threedimensional supramolecular framework through $\mathrm{N}-\mathrm{H} \cdots \mathrm{O}$, $\mathrm{O}-\mathrm{H} \cdots \mathrm{Cl}$ and $\mathrm{C}-\mathrm{H} \cdots \mathrm{Cl}$ hydrogen bonds (Table 2, Fig. 3).

\section{Hirshfeld surface analysis}

The Hirshfeld surface (Wolff et al., 2012) mapped with a $d_{\text {norm }}$ function for the asymmetric unit for the title compounds clearly shows the red spots derived from $\mathrm{H} \cdots \mathrm{O}$ and $\mathrm{H} \cdots \mathrm{Cl}$ / $\mathrm{Cl} \cdot \mathrm{H}$ contacts (Fig. 4). The two-dimensional fingerprint plot shows that the $\mathrm{H} \cdots \mathrm{Cl} / \mathrm{Cl} \cdot \mathrm{H}$ contacts associated with $\mathrm{O}-$ $\mathrm{H} \cdot \mathrm{Cl}$ hydrogen bonding appear to be the major contributor in the crystal packing (55.8\%): these contacts are represented as regions in the top left $\left(d_{\mathrm{e}}>d_{\mathrm{i}}, \mathrm{Cl} \cdots \mathrm{H}\right)$ and bottom right $\left(d_{\mathrm{e}}<d_{\mathrm{i}}, \mathrm{H} \cdots \mathrm{Cl}\right)$ of the related plots in Fig. 5. Interactions of

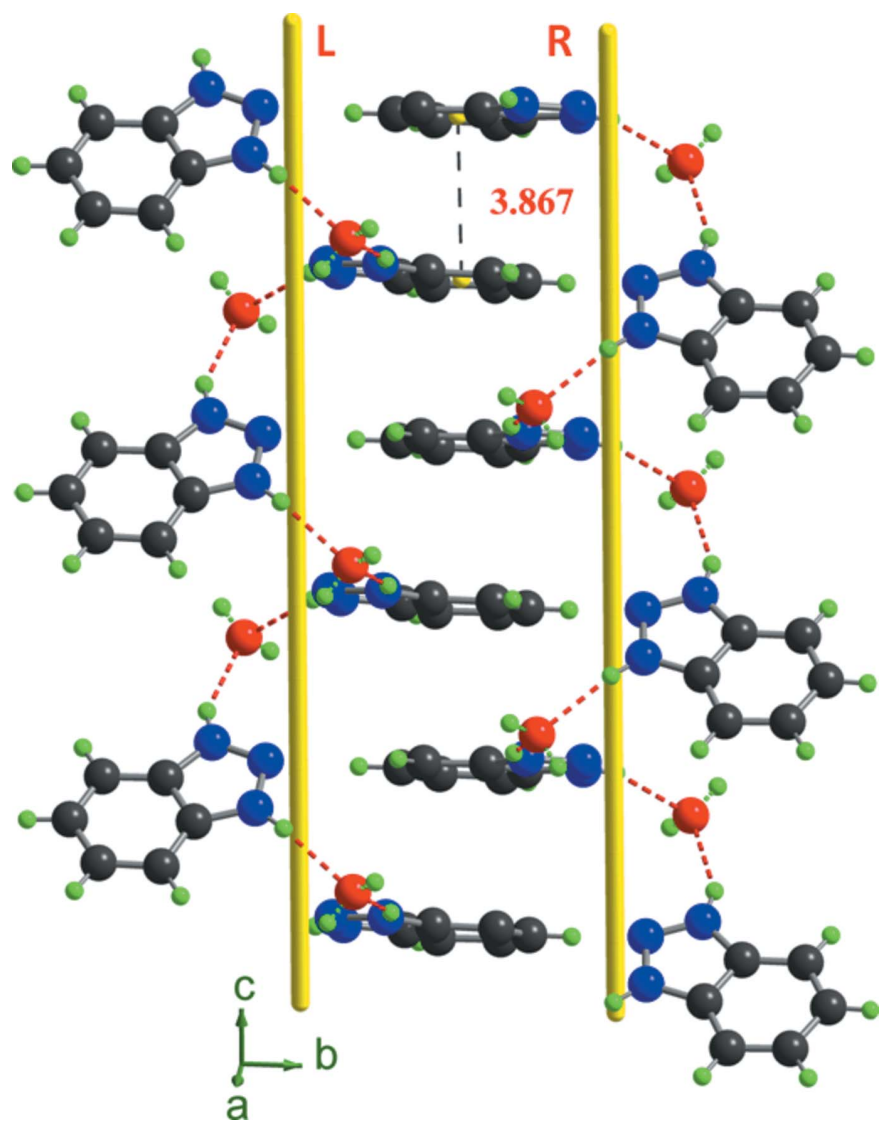

Figure 3

View of the infinite helical hydrogen-bonded chain in (I).



Figure 4

Hirshfeld surface mapped over $d_{\text {norm }}$ of (I).

the type $\mathrm{H} \cdots \mathrm{H}$ appear in the middle of the scattered points in the fingerprint maps; they comprise $10.9 \%$ of the entire surface. The decomposition of the fingerprint plot shows that $\mathrm{N} \cdots \mathrm{H} / \mathrm{H} \cdots \mathrm{N}, \mathrm{C} \cdots \mathrm{H} / \mathrm{H} \cdots \mathrm{C}, \mathrm{O} \cdots \mathrm{H} / \mathrm{H} \cdots \mathrm{O}$ and $\mathrm{N} \cdots \mathrm{Cl} / \mathrm{Cl} \cdots \mathrm{N}$ contacts have percentage contributions of $7.8 \%, 6.5 \%, 4.5 \%$ and $4.3 \%$ respectively, of the total Hirshfeld surface. The C. . C contacts associated with $\pi-\pi$ interactions amount to $3.4 \%$ of the surface: their presence is indicated by the appearance of red and blue triangles on the shape-indexed surfaces in Fig. 6. The $\mathrm{Cl} \cdots \mathrm{Bi} / \mathrm{Bi} \cdot \mathrm{Cl}(3 \%)$ interactions are represented as points in the top area. The $\mathrm{Cl} \cdots \mathrm{Cl}, \mathrm{C} \cdots \mathrm{Cl} /$ $\mathrm{Cl} \cdots \mathrm{C}, \mathrm{C} \cdots \mathrm{N}$, and $\mathrm{N} \cdots \mathrm{N}$ interactions are in the middle of the fingerprint plots, and comprise a very small contribution of $1.3 \%, 1.2 \%, 0.9 \%$ and $0.4 \%$, respectively.

The intermolecular interactions were further evaluated by using the enrichment ratio (ER; Jelsch et al., 2014). The largest contribution to the Hirshfeld surface is from $\mathrm{H} \cdots \mathrm{Cl} / \mathrm{Cl} \cdots \mathrm{H}$ contacts associated with $\mathrm{O}-\mathrm{H} \cdots \mathrm{Cl}$ hydrogen bonds and their ER value is 1.73 . The $\mathrm{H} \cdot \mathrm{H}$ contacts are the second largest contributor, but they display an enrichment ratio significantly below unity $\left(\mathrm{ER}_{\mathrm{HH}}=0.47\right)$. The formation of extensive $\pi-\pi$ interactions is reflected in the relatively high $\mathrm{ER}_{\mathrm{CC}}$ of 3.94 .

\section{Synthesis and crystallization}

The title compound was prepared by dropwise addition of an ethanolic solution of $1 H$-benzotriazole $(0.061 \mathrm{~g}, 0.5 \mathrm{mmol})$ to 

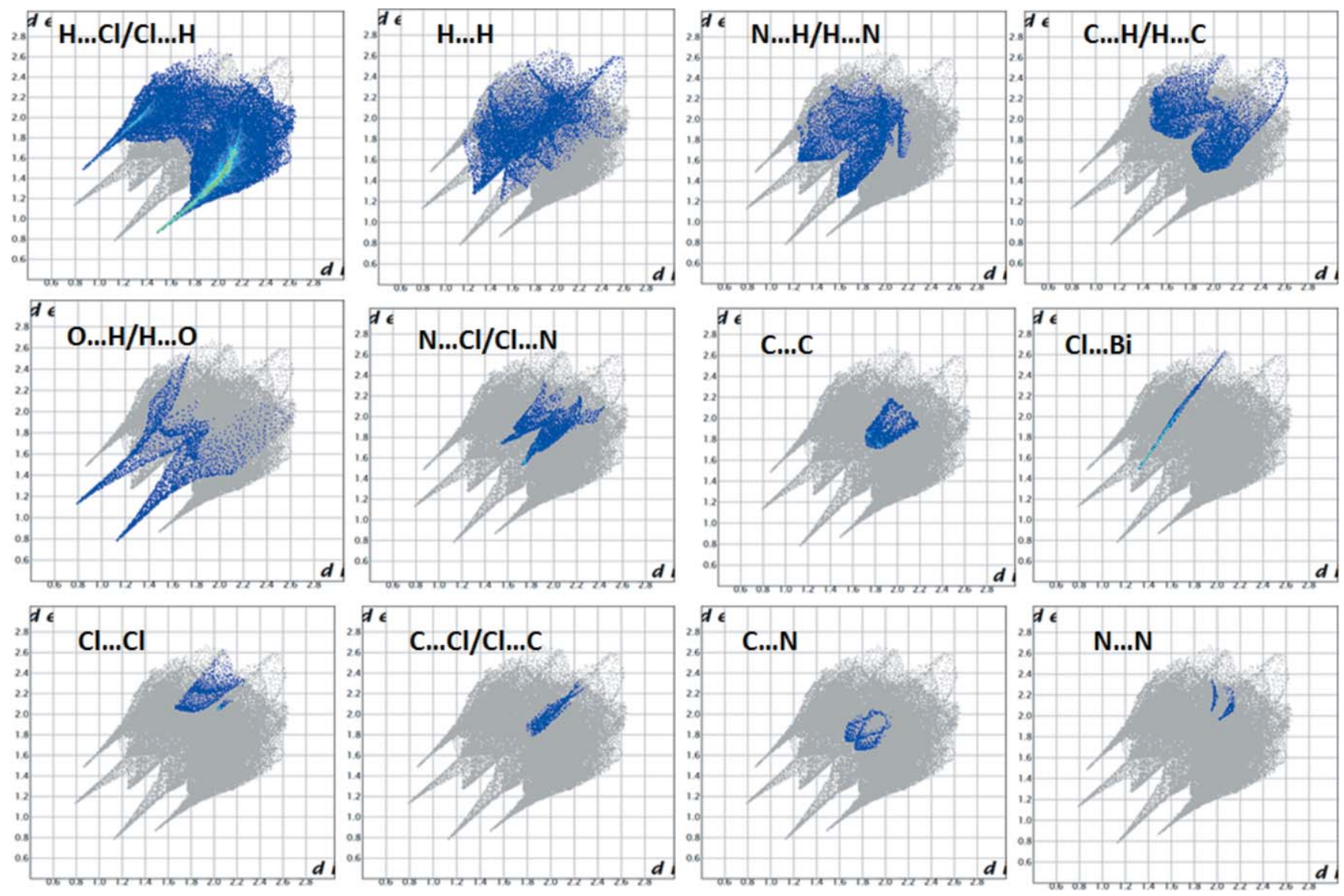

Figure 5

Two-dimensional fingerprint plots for (I) showing contributions from different contacts.

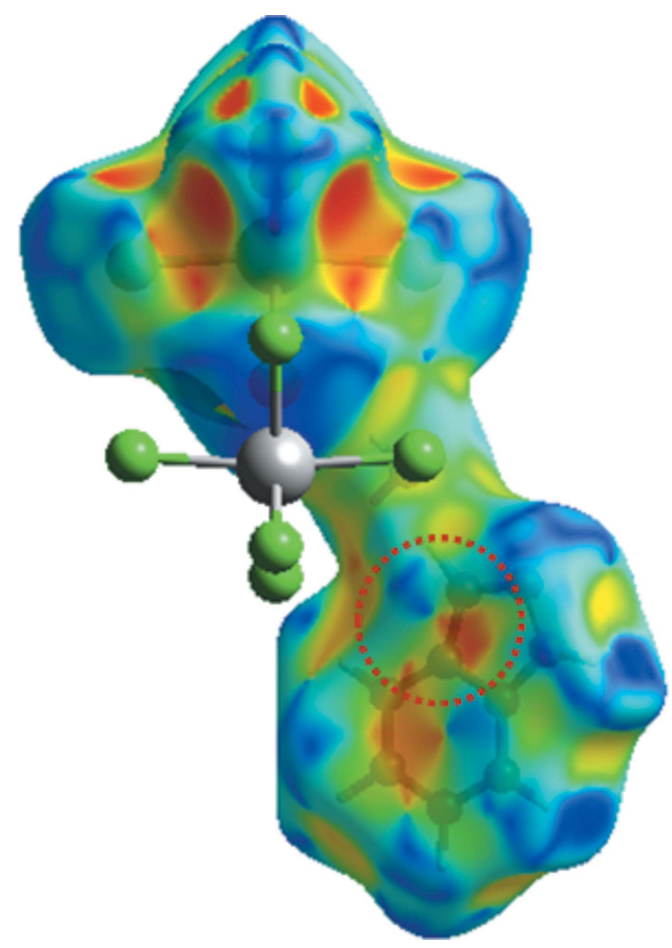

Figure 6

Hirshfeld surface mapped over the shape index for (I) highlighting the regions involved in $\pi-\pi$ stacking interactions.
$1 \mathrm{mmol}$ of a bismuth nitrate solution $\left[\mathrm{Bi}\left(\mathrm{NO}_{3}\right)_{3} \cdot 5 \mathrm{H}_{2} \mathrm{O}\right.$ ], dissolved in $0.05 \mathrm{~mL}$ of a concentrated $\mathrm{HCl}$ aqueous solution. The resulting aqueous solution was stirred for $30 \mathrm{~min}$. and kept at room temperature for crystallization. After two week of slow evaporation, colourless single crystals of (I) (yield = $75 \%$ ) were formed in the solution. Analysis observed (calculated) for $\left[\mathrm{C}_{6} \mathrm{H}_{6} \mathrm{~N}_{3}\right]_{2}\left[\mathrm{BiCl}_{5}\right] \cdot 2 \mathrm{H}_{2} \mathrm{O}(\%)$ : C 21.6 (21.0), H 2.66 (2.41), N 34.6 (33.8).

\section{Refinement}

Crystal data, data collection and structure refinement details are summarized in Table 3. The N-bound and C-bound hydrogen atoms were positioned geometrically and treated as riding: $\mathrm{N}-\mathrm{H}=0.86 \AA$ and $\mathrm{C}-\mathrm{H}=0.93 \AA$ with $U_{\text {iso }}(\mathrm{H})=$ $1.2 U_{\text {eq }}(\mathrm{N}, \mathrm{C})$. The $\mathrm{O}-\mathrm{H}$ and $\mathrm{H} \cdots \mathrm{H}$ separations in the water molecule were restrained using a DFIX model to be 0.90 and $1.46 \AA$, respectively, and refined with $U_{\text {iso }}(\mathrm{H})=1.5 U_{\text {eq }}(\mathrm{O})$.

\section{Acknowledgements}

This work was supported by the Tunisian Ministry of High Education Scientific Research. 


\section{References}

Aloui, Z., Ferretti, V., Abid, S., Lefebvre, F., Rzaigui, M. \& Nasr, C. B. (2015). J. Mol. Struct. 1097, 166-170.

Bator, G., Baran, J., Jakubas, R. \& Sobczyk, L. (1998). J. Mol. Struct. 450, 89-100.

Bator, G., Provoost, R., Silverans, R. E. \& Zeegers-Huyskens, Th. (1997). J. Mol. Struct. 435, 1-10.

Bednarska-Bolek, B., Zaleski, J., Bator, G. \& Jakubas, R. (2000). J. Phys. Chem. Solids, 61, 1249-1261.

Belter, R. K. \& Fronczek, F. R. (2013). Acta Cryst. E69, o606-0607.

Blessing, R. H. (1995). Acta Cryst. A51, 33-38.

Burnett, M. N. \& Johnson, C. K. (1996). ORTEPIII. Report ORNL6895. Oak Ridge National Laboratory, Tennessee, USA.Dammak, H., Feki, H., Boughzala, H. \& Abid, Y. (2015). Spectrochim. Acta A Mol. Biomol. Spectrosc. 137, 1235-1243.

Farrugia, L. J. (2012). J. Appl. Cryst. 45, 849-854.

Elfaleh, N. \& Kamoun, S. (2014). J. Mol. Struct. 1075, 479-485.

Ferjani, H., Boughzala, H. \& Driss, A. (2012). Acta Cryst. E68, m615.

Flack, H. D. (1983). Acta Cryst. A39, 876-881.

Jakubas, R. (1986). Solid State Commun. 60, 389-391.

Jakubas, R., Bator, G. \& Mróz, J. (1995). Acta Phys. Pol. A, 87, $663-$ 669.

Jakubas, R., Krzewska, U., Bator, G. \& Sobczyk, L. (1988). Ferroelectrics, 77, 129-135.

Jelsch, C., Ejsmont, K. \& Huder, L. (2014). IUCrJ, 1, 119-128.

Nonius (1997). KappaCCD Server Software for Windows. Nonius BV, Delft, The Netherlands.

Otwinowski, Z. \& Minor, W. (1997). Methods in Enzymology, Vol. 276, Macromolecular Crystallography, Part A, edited by C. W. Carter Jr \& R. M. Sweet, pp. 307-326. New York: Academic Press.

Ouasri, A., Jeghnou, H., Rhandour, A. \& Roussel, P. (2013). J. Solid State Chem. 200, 22-29.

Sheldrick, G. M. (2008). Acta Cryst. A64, 112-122.

Sobczyk, L., Jakubas, R. \& Zaleski, J. (1997). Pol. J. Chem. 71, 265300.

Thirunavukkarasu, A., Silambarasan, A., Chakkaravarthi, G., Mohankumar, R. \& Umarani, P. R. (2013). Acta Cryst. E69, o1605.

Wang, Y., Shi, C. \& Han, X. (2017). Polyhedron, 133, 132-136.
Table 3

Experimental details.

\begin{tabular}{|c|c|}
\hline \multicolumn{2}{|l|}{ Crystal data } \\
\hline Chemical formula & $\left(\mathrm{C}_{6} \mathrm{H}_{6} \mathrm{~N}_{3}\right)_{2}\left[\mathrm{BiCl}_{5}\right] \cdot 2 \mathrm{H}_{2} \mathrm{O}$ \\
\hline$M_{\mathrm{r}}$ & 662.54 \\
\hline Crystal system, space group & Orthorhombic, $C m c 2_{1}$ \\
\hline Temperature (K) & 293 \\
\hline$a, b, c(\AA)$ & $19.4627(4), 13.8181(4), 7.7343(2)$ \\
\hline$V\left(\AA^{3}\right)$ & $2080.04(9)$ \\
\hline$Z$ & 4 \\
\hline Radiation type & Мo $K \alpha$ \\
\hline$\mu\left(\mathrm{mm}^{-1}\right)$ & 9.14 \\
\hline Crystal size $(\mathrm{mm})$ & $0.55 \times 0.34 \times 0.23$ \\
\hline \multicolumn{2}{|l|}{ Data collection } \\
\hline Diffractometer & Nonius KappaCCD \\
\hline Absorption correction & $\begin{array}{l}\text { Multi-scan (SORTAV; Blessing, } \\
\text { 1995) }\end{array}$ \\
\hline$T_{\min }, T_{\max }$ & $0.011,0.053$ \\
\hline $\begin{array}{l}\text { No. of measured, independent and } \\
\text { observed }[I>2 \sigma(I)] \text { reflections }\end{array}$ & $6050,1670,1643$ \\
\hline$R_{\text {int }}$ & 0.067 \\
\hline$(\sin \theta / \lambda)_{\max }\left(\AA^{-1}\right)$ & 0.581 \\
\hline \multicolumn{2}{|l|}{ Refinement } \\
\hline$R\left[F^{2}>2 \sigma\left(F^{2}\right)\right], w R\left(F^{2}\right), S$ & $0.038,0.096,1.11$ \\
\hline No. of reflections & 1670 \\
\hline No. of parameters & 131 \\
\hline No. of restraints & 4 \\
\hline $\mathrm{H}$-atom treatment & $\begin{array}{l}\mathrm{H} \text { atoms treated by a mixture of } \\
\text { independent and constrained } \\
\text { refinement }\end{array}$ \\
\hline$\Delta \rho_{\max }, \Delta \rho_{\min }\left(\mathrm{e} \AA^{-3}\right)$ & $1.68,-0.76$ \\
\hline Absolute structure & Flack (1983), 731 Friedel pairs \\
\hline Absolute structure parameter & $-0.036(14)$ \\
\hline
\end{tabular}

Computer programs: Kappa CCD server software (Nonius, 1997), DENZO-SMN (Otwinowski \& Minor, 1997), SHELXS97, SHELXL97 and SHELXTL (Sheldrick, 2008), ORTEPIII (Burnett \& Johnson, 1996), and WinGX (Farrugia, 2012).

Wolff, S. K., Grimwood, D. J., McKinnon, J. J., Turner, M. J., Jayatilaka, D. \& Spackman, M. A. (2012). CrystalExplorer. University of Western Australia.

Zeng, B., Li, J. \& Wang, G. (2011). Acta Cryst. E67, o1464. 


\section{supporting information}

Acta Cryst. (2017). E73, 1759-1763 [https://doi.org/10.1107/S2056989017015134]

Synthesis, crystal structure and Hirshfeld surface analysis of a polymeric bismuthate(III) halide complex, $\left(\mathrm{C}_{6} \mathrm{H}_{6} \mathrm{~N}_{3}\right)_{2}\left[\mathrm{BiCl}_{5}\right] \cdot 2 \mathrm{H}_{2} \mathrm{O}$

\section{Chaima Boukoum, Zouhaier Aloui, Valeria Ferretti and Sonia Abid}

Computing details

Data collection: Kappa CCD server software (Nonius, 1997); cell refinement: DENZO-SMN (Otwinowski \& Minor, 1997); data reduction: DENZO-SMN (Otwinowski \& Minor, 1997); program(s) used to solve structure: SHELXS97 (Sheldrick, 2008); program(s) used to refine structure: SHELXL97 (Sheldrick, 2008); molecular graphics: ORTEPIII (Burnett \& Johnson, 1996); software used to prepare material for publication: SHELXTL (Sheldrick, 2008) and WinGX (Farrugia, 2012).

catena-Poly[bis(1,2,3-benzotriazolium) [[tetrachloridobismuth(III)]- $\mu$-chlorido] dihydrate]

Crystal data

$\left(\mathrm{C}_{6} \mathrm{H}_{6} \mathrm{~N}_{3}\right)_{2}\left[\mathrm{BiCl}_{5}\right] \cdot 2 \mathrm{H}_{2} \mathrm{O}$

$M_{r}=662.54$

Orthorhombic, $\mathrm{Cmc2}_{1}$

Hall symbol: C $2 \mathrm{c}-2$

$a=19.4627(4) \AA$

$b=13.8181$ (4) $\AA$

$c=7.7343(2) \AA$

$V=2080.04(9) \AA^{3}$

$Z=4$

$F(000)=1256$

$D_{\mathrm{x}}=2.116 \mathrm{Mg} \mathrm{m}^{-3}$

Mo $K \alpha$ radiation, $\lambda=0.71073 \AA$

Cell parameters from 8027 reflections

$\theta=4.4-7.3^{\circ}$

$\mu=9.14 \mathrm{~mm}^{-1}$

$T=293 \mathrm{~K}$

Rod, colourless

$0.55 \times 0.34 \times 0.23 \mathrm{~mm}$

Data collection

Nonius KappaCCD diffractometer

Radiation source: fine-focus sealed tube

Graphite monochromator

f scans and w scans

Absorption correction: multi-scan (SORTAV; Blessing, 1995)

$T_{\min }=0.011, T_{\max }=0.053$

6050 measured reflections

1670 independent reflections

1643 reflections with $I>2 \sigma(I)$

$R_{\text {int }}=0.067$

$\theta_{\max }=24.4^{\circ}, \theta_{\min }=4.4^{\circ}$

$h=-22 \rightarrow 22$

$k=-16 \rightarrow 16$

$l=-8 \rightarrow 8$

Refinement

Refinement on $F^{2}$

Least-squares matrix: full

$R\left[F^{2}>2 \sigma\left(F^{2}\right)\right]=0.038$

$w R\left(F^{2}\right)=0.096$

$S=1.11$

1670 reflections

131 parameters

4 restraints

Primary atom site location: structure-invariant direct methods

Secondary atom site location: difference Fourier map

Hydrogen site location: inferred from neighbouring sites

$\mathrm{H}$ atoms treated by a mixture of independent and constrained refinement 
$w=1 /\left[\sigma^{2}\left(F_{\mathrm{o}}^{2}\right)+(0.0704 P)^{2}+2.8002 P\right]$

where $P=\left(F_{\mathrm{o}}{ }^{2}+2 F_{\mathrm{c}}{ }^{2}\right) / 3$

$(\Delta / \sigma)_{\max }=0.038$

$\Delta \rho_{\max }=1.68$ e $\AA^{-3}$

$\Delta \rho_{\min }=-0.76$ e $\AA^{-3}$
Extinction correction: SHELXL97 (Sheldrick, 2008), $\mathrm{Fc}^{*}=\mathrm{kFc}\left[1+0.001 \mathrm{xFc}^{2} \lambda^{3} / \sin (2 \theta)\right]^{-1 / 4}$

Extinction coefficient: 0.0028 (4)

Absolute structure: Flack (1983), 731 Friedel pairs

Absolute structure parameter: -0.036 (14)

\section{Special details}

Geometry. All esds (except the esd in the dihedral angle between two 1.s. planes) are estimated using the full covariance matrix. The cell esds are taken into account individually in the estimation of esds in distances, angles and torsion angles; correlations between esds in cell parameters are only used when they are defined by crystal symmetry. An approximate (isotropic) treatment of cell esds is used for estimating esds involving l.s. planes.

Refinement. Refinement of $\mathrm{F}^{2}$ against ALL reflections. The weighted R-factor wR and goodness of fit $\mathrm{S}$ are based on $\mathrm{F}^{2}$, conventional R-factors $R$ are based on $F$, with $F$ set to zero for negative $F^{2}$. The threshold expression of $F^{2}>2 \operatorname{sigma}\left(\mathrm{F}^{2}\right)$ is used only for calculating R-factors(gt) etc. and is not relevant to the choice of reflections for refinement. R-factors based on $\mathrm{F}^{2}$ are statistically about twice as large as those based on F, and R-factors based on ALL data will be even larger.

Fractional atomic coordinates and isotropic or equivalent isotropic displacement parameters $\left(\AA^{2}\right)$

\begin{tabular}{lllll}
\hline & $x$ & $y$ & $z$ & $U_{\text {iso }} / U_{\mathrm{eq}}$ \\
\hline Bi1 & 0.5000 & $0.35812(2)$ & $0.08277(6)$ & $0.0310(2)$ \\
C11 & $0.36329(14)$ & $0.3716(2)$ & $0.0695(12)$ & $0.0685(10)$ \\
C12 & 0.5000 & $0.2105(2)$ & $-0.1139(6)$ & $0.0458(7)$ \\
C13 & 0.5000 & $0.2501(3)$ & $0.3697(5)$ & $0.0500(8)$ \\
C14 & 0.5000 & $0.5302(3)$ & $0.2874(5)$ & $0.0652(10)$ \\
O1W & $0.6246(4)$ & $0.3531(5)$ & $0.585(3)$ & $0.0570(18)$ \\
C1 & $0.6621(5)$ & $0.0963(7)$ & $0.7492(14)$ & $0.042(2)$ \\
C2 & $0.6058(5)$ & $0.0522(8)$ & $0.6684(15)$ & $0.049(2)$ \\
H2C & 0.5704 & 0.0873 & 0.6175 & $0.058^{*}$ \\
C3 & $0.6071(6)$ & $-0.0470(8)$ & $0.6707(16)$ & $0.054(3)$ \\
H3 & 0.5705 & -0.0804 & 0.6215 & $0.065^{*}$ \\
C4 & $0.6607(6)$ & $-0.1001(8)$ & $0.7433(16)$ & $0.056(2)$ \\
H4 & 0.6590 & -0.1673 & 0.7390 & $0.067^{*}$ \\
C5 & $0.7154(7)$ & $-0.0568(8)$ & $0.8201(15)$ & $0.047(3)$ \\
H5 & 0.7511 & -0.0926 & 0.8684 & $0.057^{*}$ \\
C6 & $0.7153(6)$ & $0.0439(9)$ & $0.8229(14)$ & $0.040(2)$ \\
N3 & $0.7585(5)$ & $0.1125(8)$ & $0.8909(15)$ & $0.050(2)$ \\
H3A & 0.7954 & 0.0992 & 0.9469 & $0.060^{*}$ \\
N1 & $0.6790(5)$ & $0.1908(7)$ & $0.7770(15)$ & $0.058(2)$ \\
H1 & 0.6545 & 0.2390 & 0.7434 & $0.070^{*}$ \\
N2 & $0.7371(5)$ & $0.1995(8)$ & $0.8609(16)$ & $0.061(3)$ \\
H11 & $0.600(5)$ & $0.400(8)$ & $0.638(18)$ & $0.091^{*}$ \\
H22 & $0.595(5)$ & $0.320(10)$ & $0.520(18)$ & $0.091^{*}$ \\
& & & &
\end{tabular}

Atomic displacement parameters $\left(\AA^{2}\right)$

\begin{tabular}{lllllll}
\hline & $U^{11}$ & $U^{22}$ & $U^{33}$ & $U^{12}$ & $U^{13}$ & $U^{23}$ \\
\hline Bi1 & $0.0321(3)$ & $0.0286(3)$ & $0.0323(3)$ & 0.000 & 0.000 & $-0.0007(2)$ \\
C11 & $0.0362(11)$ & $0.0924(19)$ & $0.077(3)$ & $0.0003(11)$ & $0.008(2)$ & $-0.013(2)$ \\
C12 & $0.0575(19)$ & $0.0308(16)$ & $0.0490(17)$ & 0.000 & 0.000 & $-0.0089(14)$
\end{tabular}


supporting information

$\begin{array}{lllllll}\mathrm{Cl} 3 & 0.0605(19) & 0.0469(19) & 0.0427(16) & 0.000 & 0.000 & 0.0107(16) \\ \mathrm{C} 4 & 0.088(3) & 0.053(2) & 0.054(2) & 0.000 & 0.000 & -0.0215(16) \\ \mathrm{O} 1 \mathrm{~W} & 0.042(3) & 0.060(5) & 0.069(5) & 0.002(2) & 0.002(10) & -0.010(5) \\ \mathrm{C} 1 & 0.041(5) & 0.033(5) & 0.051(5) & 0.001(4) & 0.009(4) & -0.002(4) \\ \mathrm{C} 2 & 0.047(5) & 0.050(6) & 0.049(5) & 0.008(4) & 0.001(4) & -0.002(4) \\ \mathrm{C} 3 & 0.048(5) & 0.056(7) & 0.059(6) & -0.008(5) & -0.001(5) & -0.011(5) \\ \mathrm{C} 4 & 0.064(6) & 0.042(6) & 0.062(6) & -0.004(5) & 0.016(5) & -0.005(5) \\ \mathrm{C} 5 & 0.053(7) & 0.042(6) & 0.047(6) & 0.011(5) & 0.002(5) & 0.001(5) \\ \mathrm{C} 6 & 0.032(5) & 0.046(5) & 0.040(5) & 0.004(4) & 0.002(4) & -0.007(5) \\ \mathrm{N} 3 & 0.044(5) & 0.053(6) & 0.053(6) & -0.002(5) & 0.005(4) & -0.006(4) \\ \mathrm{N} 1 & 0.052(5) & 0.037(5) & 0.085(7) & -0.001(4) & 0.011(5) & -0.005(5) \\ \mathrm{N} 2 & 0.053(6) & 0.050(6) & 0.080(7) & -0.016(5) & 0.010(5) & -0.018(5) \\ & & & & & & \end{array}$

Geometric parameters $\left(\AA,{ }^{\circ}\right)$

\begin{tabular}{|c|c|c|c|}
\hline $\mathrm{Bi} 1-\mathrm{Cl1}$ & $2.669(3)$ & $\mathrm{C} 2-\mathrm{H} 2 \mathrm{C}$ & 0.9300 \\
\hline $\mathrm{Bi} 1-\mathrm{Cl1}^{\mathrm{i}}$ & $2.669(3)$ & $\mathrm{C} 3-\mathrm{C} 4$ & $1.394(17)$ \\
\hline $\mathrm{Bi} 1-\mathrm{Cl} 2$ & $2.545(3)$ & $\mathrm{C} 3-\mathrm{H} 3$ & 0.9300 \\
\hline $\mathrm{Bi} 1-\mathrm{Cl} 3$ & $2.674(4)$ & $\mathrm{C} 4-\mathrm{C} 5$ & $1.358(18)$ \\
\hline $\mathrm{Bi} 1-\mathrm{Cl} 4^{\mathrm{ii}}$ & $2.757(4)$ & $\mathrm{C} 4-\mathrm{H} 4$ & 0.9300 \\
\hline $\mathrm{Bi1}-\mathrm{Cl} 4$ & $2.856(4)$ & $\mathrm{C} 5-\mathrm{C} 6$ & $1.392(13)$ \\
\hline $\mathrm{Cl} 4-\mathrm{Bi} 1^{\mathrm{iii}}$ & $2.757(4)$ & $\mathrm{C} 5-\mathrm{H} 5$ & 0.9300 \\
\hline $\mathrm{O} 1 \mathrm{~W}-\mathrm{H} 11$ & $0.90(2)$ & $\mathrm{C} 6-\mathrm{N} 3$ & $1.370(15)$ \\
\hline $\mathrm{O} 1 \mathrm{~W}-\mathrm{H} 22$ & $0.90(2)$ & $\mathrm{N} 3-\mathrm{N} 2$ & $1.293(15)$ \\
\hline $\mathrm{C} 1-\mathrm{N} 1$ & $1.364(16)$ & $\mathrm{N} 3-\mathrm{H} 3 \mathrm{~A}$ & 0.8600 \\
\hline $\mathrm{C} 1-\mathrm{C} 6$ & $1.387(16)$ & $\mathrm{N} 1-\mathrm{N} 2$ & $1.308(15)$ \\
\hline $\mathrm{C} 1-\mathrm{C} 2$ & $1.402(15)$ & $\mathrm{N} 1-\mathrm{H} 1$ & 0.8600 \\
\hline $\mathrm{C} 2-\mathrm{C} 3$ & $1.371(16)$ & & \\
\hline $\mathrm{Cl} 2-\mathrm{Bi} 1-\mathrm{Cl} 1$ & $91.88(13)$ & $\mathrm{C} 3-\mathrm{C} 2-\mathrm{H} 2 \mathrm{C}$ & 122.8 \\
\hline $\mathrm{Cl} 2-\mathrm{Bi} 1-\mathrm{Cl}^{\mathrm{i}}$ & $91.88(13)$ & $\mathrm{C} 1-\mathrm{C} 2-\mathrm{H} 2 \mathrm{C}$ & 122.8 \\
\hline $\mathrm{C} 11-\mathrm{Bi} 1-\mathrm{Cl1}{ }^{\mathrm{i}}$ & $170.9(2)$ & $\mathrm{C} 2-\mathrm{C} 3-\mathrm{C} 4$ & $123.0(10)$ \\
\hline $\mathrm{Cl} 2-\mathrm{Bi} 1-\mathrm{Cl} 3$ & $92.80(16)$ & $\mathrm{C} 2-\mathrm{C} 3-\mathrm{H} 3$ & 118.5 \\
\hline $\mathrm{C} 11-\mathrm{Bi} 1-\mathrm{Cl} 3$ & $94.06(17)$ & $\mathrm{C} 4-\mathrm{C} 3-\mathrm{H} 3$ & 118.5 \\
\hline $\mathrm{C} 11{ }^{\mathrm{i}}-\mathrm{Bi} 1-\mathrm{Cl} 3$ & $94.06(17)$ & $\mathrm{C} 5-\mathrm{C} 4-\mathrm{C} 3$ & $122.1(10)$ \\
\hline $\mathrm{Cl} 2-\mathrm{Bi} 1-\mathrm{Cl} 4^{\mathrm{ii}}$ & $87.32(14)$ & $\mathrm{C} 5-\mathrm{C} 4-\mathrm{H} 4$ & 118.9 \\
\hline $\mathrm{C} 11-\mathrm{Bi} 1-\mathrm{Cl} 4^{\mathrm{ii}}$ & $85.93(17)$ & $\mathrm{C} 3-\mathrm{C} 4-\mathrm{H} 4$ & 118.9 \\
\hline $\mathrm{Cl} 1^{\mathrm{i}}-\mathrm{Bi} 1-\mathrm{Cl} 4^{\mathrm{ii}}$ & $85.93(17)$ & $\mathrm{C} 4-\mathrm{C} 5-\mathrm{C} 6$ & $116.5(12)$ \\
\hline $\mathrm{Cl} 3-\mathrm{Bi} 1-\mathrm{Cl}^{\mathrm{ii}}$ & $179.88(13)$ & $\mathrm{C} 4-\mathrm{C} 5-\mathrm{H} 5$ & 121.8 \\
\hline $\mathrm{Cl} 2-\mathrm{Bi} 1-\mathrm{Cl} 4$ & $176.93(13)$ & $\mathrm{C} 6-\mathrm{C} 5-\mathrm{H} 5$ & 121.8 \\
\hline $\mathrm{Cl1}-\mathrm{Bi1}-\mathrm{Cl} 4$ & $87.90(13)$ & $\mathrm{N} 3-\mathrm{C} 6-\mathrm{C} 1$ & $104.7(10)$ \\
\hline $\mathrm{Cl1}{ }^{\mathrm{i}}-\mathrm{Bi} 1-\mathrm{Cl} 4$ & $87.90(13)$ & $\mathrm{N} 3-\mathrm{C} 6-\mathrm{C} 5$ & $134.1(13)$ \\
\hline $\mathrm{Cl} 3-\mathrm{Bi} 1-\mathrm{Cl} 4$ & $90.27(13)$ & $\mathrm{C} 1-\mathrm{C} 6-\mathrm{C} 5$ & $121.1(13)$ \\
\hline $\mathrm{Cl} 4^{\mathrm{ii}}-\mathrm{Bi} 1-\mathrm{Cl} 4$ & $89.61(3)$ & $\mathrm{N} 2-\mathrm{N} 3-\mathrm{C} 6$ & $112.1(10)$ \\
\hline $\mathrm{Bi} 1^{i \mathrm{ii}}-\mathrm{Cl} 4-\mathrm{Bi} 1$ & $157.68(19)$ & $\mathrm{N} 2-\mathrm{N} 3-\mathrm{H} 3 \mathrm{~A}$ & 123.9 \\
\hline $\mathrm{H} 11-\mathrm{O} 1 \mathrm{~W}-\mathrm{H} 22$ & $106(3)$ & $\mathrm{C} 6-\mathrm{N} 3-\mathrm{H} 3 \mathrm{~A}$ & 123.9 \\
\hline $\mathrm{N} 1-\mathrm{C} 1-\mathrm{C} 6$ & $104.8(10)$ & $\mathrm{N} 2-\mathrm{N} 1-\mathrm{C} 1$ & $112.0(10)$ \\
\hline $\mathrm{N} 1-\mathrm{C} 1-\mathrm{C} 2$ & $132.5(10)$ & $\mathrm{N} 2-\mathrm{N} 1-\mathrm{H} 1$ & 124.0 \\
\hline
\end{tabular}




$\begin{array}{llll}\mathrm{C} 6-\mathrm{C} 1-\mathrm{C} 2 & 122.7(10) & \mathrm{C} 1-\mathrm{N} 1-\mathrm{H} 1 & 124.0 \\ \mathrm{C} 3-\mathrm{C} 2-\mathrm{C} 1 & 114.5(10) & \mathrm{N} 3-\mathrm{N} 2-\mathrm{N} 1 & 106.4(9)\end{array}$

Symmetry codes: (i) $-x+1, y, z$; (ii) $-x+1,-y+1, z-1 / 2$; (iii) $-x+1,-y+1, z+1 / 2$.

Hydrogen-bond geometry $\left(A,{ }^{\circ}\right)$

\begin{tabular}{lllll}
\hline$D-\mathrm{H} \cdots A$ & $D-\mathrm{H}$ & $\mathrm{H} \cdots A$ & $D \cdots A$ & $D-\mathrm{H} \cdots A$ \\
\hline $\mathrm{N} 1-\mathrm{H} 1 \cdots \mathrm{O} 1 W$ & 0.86 & 2.08 & $2.891(17)$ & 157 \\
$\mathrm{~N} 3-\mathrm{H} 3 A \cdots \mathrm{O} 1 W^{\text {iv }}$ & 0.86 & $2.00(2)$ & $2.767(18)$ & 148 \\
$\mathrm{O} 1 W-\mathrm{H} 11 \cdots \mathrm{Cl} 4^{\mathrm{iii}}$ & $0.89(11)$ & $2.47(11)$ & $3.306(13)$ & $157(9)$ \\
$\mathrm{O} 1 W-\mathrm{H} 22 \cdots \mathrm{Cl} 3$ & $0.90(12)$ & $2.38(12)$ & $3.268(14)$ & $169(11)$ \\
$\mathrm{C} 5-\mathrm{H} 5 \cdots \mathrm{C} 11^{v}$ & 0.93 & 2.73 & $3.603(14)$ & 157
\end{tabular}

Symmetry codes: (iii) $-x+1,-y+1, z+1 / 2$; (iv) $-x+3 / 2,-y+1 / 2, z+1 / 2$; (v) $x+1 / 2, y-1 / 2, z+1$. 